\title{
Efecto del contexto verbal en el reconocimiento de la expresión de alegría y tristeza
}

\section{Effect of verbal context on the expression of happiness and sadness}

\author{
Fernando Gordillo ${ }^{1}$, Miguel Ángel Pérez ${ }^{1}$, Gabriela Castillo ${ }^{1}$, Lilia Mestas ${ }^{2}$, \\ José María Arana ${ }^{3}$ y Rafael Manuel López ${ }^{4}$ \\ ${ }^{1}$ Departamento de Psicología, Universidad Camilo José Cela, Madrid, España. \\ ${ }^{2}$ Facultad de Estudios Superiores Zaragoza. Universidad Nacional Autónoma de México, México. \\ ${ }^{3}$ Departamento de Psicología, Universidad de Salamanca, España. \\ ${ }^{4}$ Nonverbal Behavior Analysis Group - Behavior \& Law Research Foundation, Madrid, España.
}

Disponible online 30 de abril de 2016

\begin{abstract}
El reconocimiento de las emociones supone la integración de los aspectos físicos relativos a los movimientos de la musculatura facial, pero también y desde diferentes enfoques se da relevancia al contexto que rodea a dicha expresión en el momento de ser percibida. En la presente investigación se analizó el papel del contexto verbal sobre el reconocimiento de la expresión facial de alegría y tristeza. Participaron 50 sujetos a los que se les pidió que visualizaran expresiones mixtas neutras rodeadas por cinco palabras positivas, negativas o neutras (contexto verbal), que describían la vida de dicha persona (fase I). Posteriormente se les presentaron durante 39 milisegundos expresiones faciales mixtas de alegría o tristeza de las mismas personas que aparecieron en la fase anterior para que decidieran lo más rápido posible si la emoción expresada era de alegría o tristeza (fase II). Los resultados mostraron un peor reconocimiento de la expresión de tristeza respecto a la de alegría cuando el contexto verbal previo era positivo. Por otro lado, la expresión de tristeza se reconoció mejor cuando iba precedida de un contexto verbal negativo respecto a cuando era positivo. Se discuten los resultados dentro del modelo constructivista de la emoción.
\end{abstract}

Palabras Clave: Procesamiento Automático; Emoción; Contexto verbal; Modelo Constructivista; Percepción.

The recognition of emotions involves a combination of physical aspects related to movements of the facial muscles. In addition, different approaches have drawn attention to the context in which facial expressions occur when they are being perceived. This study analysed the role of verbal context in the recognition of the facial expressions of happiness and sadness. The study included 50 participants. They were asked to view images of mixed neutral expressions surrounded by five positive, negative, or neutral words (verbal context) that described the life an individual (Phase I). Following this, they were shown happy or sad facial expressions of the same individuals who appeared in the previous phase. The images were displayed for $39 \mathrm{~ms}$ such that the participants had to decide as quickly as possible whether the expression was happy or sad (Phase II). The results showed that participants found expressions of sadness harder to recognise than expressions of happiness when the former were preceded by a positive verbal context. In contrast, they found that expressions of sadness were easier to recognise than expressions of happiness when the former were preceded by a negative verbal context. The results are discussed within the constructivist model of emotion.

Key Words: Automatic Processing; Emotion; Verbal context; Constructivist Model; Perception.

Correspondencia: Fernando Gordillo. Universidad Camilo José Cela, Departamento de Psicología. Castillo de Alarcón nº 49. 28692-Villafranca del Castillo (Madrid). E-mail: fgordillo@ucjc.edu / fgordilloleon@hotmail.com. E-mails de los co-autores: Miguel Ángel Pérez, mperez@ucjc.edu; Gabriela Castillo, gcastillo@ucjc.edu; Lilia Mestas, lilia_mestas@yahoo.com.mx; José M. Arana, arana@usal.es y, Rafael Manuel López, rlopez@ behaviorandlaw.com. 
Las teorías clásicas de la emoción consideran que las señales emocionales, entre ellas las que provienen de las expresiones faciales, tienen un componente universal e innato que requiere de un mínimo procesamiento conceptual de la información para ser reconocidas (Ekman y Cordaro, 2011). Sin embargo, desde el enfoque constructivista, se considera que la percepción visual no se produce de manera pasiva y automática y que las personas "construyen" y participan en la percepción de aquello que están viendo a través de sus motivaciones, expectativas o experiencias previas (Bruner, 1957; Bruner, Postman y Rodrigues, 1951). Llevando esta idea al ámbito de las emociones, se considera que la expresión facial emocional no es una señal discreta que de manera automática el perceptor incluye en una u otra categoría, sino que es una señal ambigua que informa sobre los niveles de valencia y activación, y que es a través del conocimiento conceptual que los sujetos tienen de las emociones como pueden incluirlas en una $u$ otra categoría emocional (Barrett, Lindquist y Gendron, 2007; Russell, 2003; Russell, Bachorowski y Fernández-Dols, 2003).

Por lo tanto, desde esta perspectiva, la expresión facial es considerada una señal de comunicación dinámica que en pocas ocasiones adquiere significado de manera aislada (Shen-Mou y Lee-Xieng, 2013), sino que requiere de un contexto que podría estar afectando a su percepción e interpretación (Wieser y Brosch, 2012). Este enfoque constructivista ha encontrado apoyo experimental desde los estudios clásicos de Russel sobre emoción y percepción (e.g., Russel, 1994), hasta recientes investigaciones donde se informa que determinados movimientos faciales pueden incluirse en diferentes categorías emocionales (Jack, Garrod y Schyns, 2014) y que sería la cultura la que determinaría su inclusión en una u otra categoría (Jack, Garrod, Yu, Caldara y Schyns, 2012). De manera más concreta, se ha comprobado que el contexto que rodea a una expresión facial afecta a la interpretación de la emoción percibida (e.g., Aviezer et al., 2008; Hassin, Aviezer y Bentin, 2013).

Respecto al Contexto Verbal $(\mathrm{Cv})$, se puede entender como la información que el perceptor conoce de la persona emisora de la Expresión Facial (Ef). En este sentido y dentro del campo de la emoción, el modelo constructivista considera que el $\mathrm{Cv}$ incrementaría el acceso al conocimiento conceptual afectando al juicio realizado sobre la emoción expresada, incluso más que los movimientos musculares de la cara (Barrett y Kensinger, 2010; Barrett et al., 2007). El Cv tendría un papel determinante en la valoración y reconocimiento de las emociones (Hassin et al., 2013). Este planteamiento ha sido respaldado por estudios de neuroimagen (Wager et al., 2008), donde estructuras como el giro frontal inferior, que forma parte de la red neuronal encargada de la percepción de las emociones, participaría también en el lenguaje (Gitelman, Nobre, Sonty, Parrish y Mesulam, 2005) y en la recuperación del conocimiento conceptual (Badre, Poldrack, Paré-Bagoev, Isler y Wagner, 2005).
Se puede establecer una distinción entre aquellos trabajos que han estudiado el $\mathrm{Cv}$ en términos de etiquetas verbales ("palabra"), y los que han utilizado descripciones de situaciones sociales ("frase/s"). Respecto a los primeros se ha comprobado que una palabra puede afectar a la valoración y reconocimiento posterior de las expresiones faciales emocionales (Lindquist, Barrett, Bliss-Moreau y Russell, 2006). Por otro lado, la presentación de una frase negativa ("perdió 500 dólares") versus positiva ("ganó 500 dólares"), de manera previa a la visualización de una expresión de sorpresa, permitió modular la activación de la amígdala en respuesta a dicha expresión (Kim et al., 2004). De igual manera, y ante expresiones neutras, la presentación previa de una frase referida al perceptor ("esta persona piensa que eres competente / incompetente"), tuvo efectos significativos sobre la activación de determinadas estructuras cerebrales, y sobre la valoración de la expresión facial (Schwarz, Wieser, Gerdes, Mühlberger y Pauli, 2013).

En términos generales, el efecto encontrado resulta más fuerte cuando la expresión facial utilizada es neutra o ambigua. Además, la mayoría de las evidencias muestran que cuando $\mathrm{Cv}$ y la expresión facial son congruentes (e.g., Cv Positivo - Expresión de alegría), se produce una facilitación en el reconocimiento y valoración de dicha expresión (Matsumoto y Hwang, 2010; Wieser y Brosch, 2012). De estos trabajos se desprende la importancia no solo de la valencia emocional de la expresión facial y del $\mathrm{Cv}$, también de la relación de congruencia o incongruencia que se establezca entre ellas. Sin embargo, hasta la fecha no se ha estudiado con la suficiente profundidad el $\mathrm{CV}$ entendido como el conocimiento que el perceptor tiene de la vida de la persona que expresa la emoción (Gordillo, Lozano, López, Pérez, Arana y Mestas, 2013).

Para estudiar el Cv desde esta perspectiva se asume que de igual manera que las palabras y las frases pueden determinar un efecto significativo sobre la valoración de una expresión facial, también podría hacerlo una red semántica más amplia y relativa a la vida de la persona productora de la expresión facial. Desde los modelos de redes semánticas (Niedenthal, 2008), este planteamiento es posible en tanto la emoción está representada por un nodo de memoria que incluye pensamientos, creencias, metas y acciones que se asociaron en su momento a dicha emoción.

Otra cuestión de gran trascendencia, y todavía sin dilucidar, es si el efecto del Cv sobre el reconocimiento de la expresión facial se produce de manera automática. Se considera que un proceso es automático si respeta una serie de criterios, como son: ser inconsciente; no puede detenerse una vez iniciado; no requiere recursos mentales conscientes, y no es intencionado. Son muchos los trabajos que confirman el efecto prime de diferentes estímulos emocionales sobre la valoración posterior de otro estímulo (target) (e.g., Janiszewski y Wyer, 2014). En concreto, cuando el target es una expresión facial, se ha com- 
probado que el efecto de la postura corporal que enmarca la expresión facial (contexto corporal) sobre el reconocimiento de la emoción a través del rostro se produce de manera no intencional, incontrolada y con relativo poco esfuerzo (Aviezer, Bentin, Dudarev y Hassin, 2011). De igual manera se han encontrado evidencias sobre el efecto automático que la presentación breve de una etiqueta verbal tiene sobre el reconocimiento de la expresión facial emocional (Barret et al., 2007).

Esta investigación pretende analizar, por un lado, el efecto que el $\mathrm{Cv}$ referido a la vida de la persona que produce la expresión facial (positivo, neutro, negativo) tiene sobre el reconocimiento de la emoción expresada (alegría, tristeza). Por otro lado, se pretende analizar si este proceso se produce de manera automática. Estos objetivos requieren la elaboración de un paradigma que permita la codificación de la información relativa a la vida de una persona (fase I), para que esta información se active de manera eficaz en la fase de reconocimiento (fase II).

En base al modelo constructivista de la emoción (Barrett y Kensinger, 2010; Barret, Pesquita y Gendron, 2011), donde el $\mathrm{Cv}$, como etiqueta verbal o situación social, afectaría al reconocimiento de la expresión emocional, y a partir de los trabajos consultados en la literatura científica, donde se informa de un efecto facilitador en el reconocimiento de la expresión facial cuando el contexto es congruente (véase Matsumoto y Hwang, 2010; Wieser y Brosch, 2012); se espera que el $\mathrm{Cv}$ positivo, relativo a la vida de la persona productora de la expresión facial de lugar a un mejor reconocimiento de la expresión facial de alegría respecto a la de tristeza, y lo contrario para el Cv negativo $\left(\mathrm{H}_{1}\right)$. Por otro lado, de aceptarse esta hipótesis, y dado que para el reconocimiento de la emoción se utilizaron tiempos de exposición de la expresión facial de 39 ms, se aceptaría la automaticidad del proceso $\left(\mathrm{H}_{2}\right)$, que otros autores han encontrado para el contexto corporal (e.g., Aviezer et al., 2011), visual y referido al tipo de escena que rodea a la expresión facial en el momento de su percepción (e.g., Righart y de Gelder, 2008), y verbal en términos de etiquetas verbales (Pell, Jaywant, Montea y Kotz, 2012).

\section{Participantes \\ Método}

La muestra estuvo formada por 50 estudiantes de la Universidad Camilo José Cela, que estaban realizando el primer curso del Grado de Psicología (84 \% mujeres) con edades comprendidas entre los 18 y 31 años $(M=20.72, D T=3.09)$, y que dieron su consentimiento informado para participar voluntariamente en el experimento.

\section{Instrumentos}

Para la programación de la tarea se utilizó el software E-prime (Schneider, Eschman y Zuccolotto, 2002), y los estímulos fueron presentados en una pantalla de 15 pulgadas, a una distancia aproximada de $50 \mathrm{~cm}$. de los participantes. Se utilizaron 5 palabras con valencia positiva, 5 con valencia negativa y 10 con valencia neutra, obtenidas del estudio normativo reali- zado por Redondo, Fraga, Comesaña y Perea (2005). También se utilizaron cuatro expresiones faciales prototípicas de alegría y cuatro de tristeza a partir de cuatro modelos diferentes (dos hombres y dos mujeres), pertenecientes a la base de datos NimStimFace Stimulus Set (Tottenham et al., 2009) (Anexo 1). A partir de estas ocho expresiones faciales prototípicas se elaboraron expresiones con morphing (25\% alegría-75\% tristeza; $50 \%$ alegría- $50 \%$ tristeza; $75 \%$ alegría- $25 \%$ tristeza) utilizando el programa Magic Morph (iTinysoft, 2002). Este programa permitió transformar una fotografía en otra marcando en cada una de las imágenes los puntos de transición. La utilización de este tipo de expresiones mixtas se hizo con la intención de favorecer, gracias a su mayor ambigüedad, un posible sesgo derivado del efecto del contexto verbal. La utilización de este tipo de expresiones ha sido justificada en diferentes estudios por su mayor sensibilidad (Matsumoto y Hwang, 2010; Wieser y Brosch, 2012).

\section{Procedimiento}

A todos los participantes se les informó en horario de clase que iban a realizar una tarea de tiempo de respuesta, sin especificar el tipo de tarea que sería. También se les solicitó el consentimiento informado y se prepararon las listas con el día y la hora a la que debían presentarse en el laboratorio para realizar la tarea. Todos los participantes pasaron de uno en uno por las siguientes fases:

Fase I: codificación y valoración del contexto verbal. A los participantes se les presentó la fotografía de una expresión neutra (50\% alegría / 50\% tristeza) dentro de un círculo en el que se distribuyeron cinco palabras positivas, negativas o neutras que estaban asociadas a una edad que iba de los 5 a los 30 años. Se les informó por escrito que las palabras que verían, describían la vida de la persona que aparecía dentro del círculo. Tenían que observar este esquema durante dos minutos. Mediante este método se pretendía que la persona integrara la información de la expresión facial con la información verbal que simulaba los sucesos acontecidos en la vida de esa persona (Anexo 2). Para integrar adecuadamente esta impresión, una vez pasados los 2 minutos, se les pedía que valoraran en qué grado consideraban que la vida de esa persona era positiva o negativa (muy negativa_1.....9_muy positiva).

Con este procedimiento a cada sujeto se le presentaban cuatro expresiones faciales neutras, dos de hombre y dos de mujer, que se asociaban a dos contextos neutros, uno negativo y otro positivo Se realizó un contrabalanceo, de forma que todas las expresiones se asociaron con todos los tipos de $\mathrm{Cv}$ (positivo, neutro y negativo). Esto se consiguió haciendo que para cada sujeto variara la asociación entre expresión y tipo de contexto, hasta conseguir el mismo número de asociaciones por cada condición. De esta forma se evitaba que las peculiaridades de las expresiones faciales (estructura facial, pelo, etc.) pudieran estar afectando al proceso de reconocimiento de manera no controlada. 
Fase II: Reconocimiento de la expresión emocional. En esta segunda fase se presentaron las expresiones faciales mixtas de tristeza (25\% alegría/ $75 \%$ tristeza) y alegría (75\% alegría / $25 \%$ tristeza) de las mismas personas que aparecieron en la fase I con expresión neutra ( $50 \%$ alegría / 50\% tristeza), en total 8 fotografías. Por otro lado, las ocho fotografías de la fase II se presentaron de manera aleatoria con la siguiente secuencia: primero aparecía una señal de atento $(2.000 \mathrm{~ms})$, seguida de una cruz para fijar la mirada en el centro de la pantalla (1.000 $\mathrm{ms}$ ), seguido de la presentación del objetivo (expresión facial) durante $39 \mathrm{~ms}$ y por último una pantalla en blanco durante 160 ms.

Se les dio la instrucción, por escrito y en una pantalla previa a iniciar la fase II, que les aparecerían, durante $39 \mathrm{~ms}$ cada una, expresiones faciales que podían ser de alegría o tristeza. $\mathrm{Su}$ cometido era contestar lo más rápido posible si la expresión era de alegría (pulsando "S") o tristeza (pulsando "L"). Se varió en cada sujeto la asociación entre tecla ("L", "S") y el tipo de expresión (alegría, tristeza).

\section{Análisis de los datos}

Análisis de las diferencias en la valoración del contexto verbal. Se realizó un ANOVA de medidas repetidas con la variable Contexto Verbal ( $\mathrm{Cv}$ : positivo, neutro y negativo) como variable independiente y la valoración del contexto verbal realizada en la fase I como variable dependiente.

Análisis de las diferencias en la tasa de reconocimiento y el tiempo de respuesta. Se realizaron dos ANOVA de medidas repetidas 2 x 3, con las variables Expresión Facial (Ef: alegría y tristeza) y Contexto Verbal (Cv: positivo, neutro, negativo) como variables independientes, y la tasa de reconocimiento y los tiempos de respuesta como variables dependientes (fase II).

\section{Resultados}

El ANOVA de medidas repetidas realizado con la valoración del contexto verbal (fase I) mostró un efecto significativo del $\mathrm{Cv}\left[F(2,98)=144.85, p<.001, \eta_{p}^{2}=0.86, p=1.00\right]$. $\mathrm{El}$ análisis Bonferroni evidenció que las diferencias se dieron entre todos los niveles $(p<.001)$ : $\mathrm{Cv}$ positivo $(M=6.66, D T$ $=1.67, I C$ del $95 \%: 6.18-7.14)$, neutro $(M=5.57, D T=1.15$, IC $95 \%$ : 5.24-5.90) y negativo $(M=2.44, D T=1.45$, IC 95 $\%: 2.03-2.85)$. Se evidenció que la valoración realizada al $\mathrm{Cv}$ positivo distaba 1.9 puntos de la valoración realizada al $\mathrm{Cv}$ neutro, mientras que el $\mathrm{Cv}$ negativo distaba 3.13 puntos del $\mathrm{Cv}$ neutro. Por lo tanto había un desequilibrio moderado entre las distancias relativas al $\mathrm{Cv}$ neutro que debían tenerse en cuenta a la hora de interpretar los resultados.

El ANOVA de medidas repetidas mostró un efecto significativo sobre el reconocimiento de las expresiones emocionales del $\mathrm{Cv}\left[F(2,98)=4.30, p=.016, \eta^{2}=.08, p=.74\right]$ y la $\mathrm{Ef}$ $\left[F(1,49)=9.37, p=.004, \eta_{p}^{2}=.16, p=.85\right]$, así como de la interacción entre $\mathrm{Cv}$ y $\operatorname{Ef}\left[F(2,98)=7.43, p=.001, \eta_{p}^{2}=.13\right.$, $p=.94]$. El análisis Bonferroni reveló que las diferencias solo fueron significativas entre el $\mathrm{Cv}$ positivo $[M=1.80, D T=0.50$ , (95\% IC: $1,71-1,89)]$ y el Cv negativo $[M=1.93, D T=0.29$, (95\% IC: 1.87-1.99)], $\left(M_{i-j}=-0.130, S E=.049, p=.033\right)$, y que se reconocieron mejor las Ef de alegría $[M=1.92, D T=0.26$, (95\% IC: 1.88-1.96)] que las Ef de tristeza $[M=1.81, D T=$ 0.40, (95\% IC: $1.74-1.88)]$.

Respecto a la interacción, el análisis de los efectos simples (método Bonferroni para el control de la tasa de error) reveló que las diferencias en la tasa de reconocimiento entre la Ef de alegría y tristeza solo fueron significativas dentro del $\mathrm{Cv}$ positivo y favorables a la Ef de alegría.

Por otro lado, solo se mostraron diferencias entre el $\mathrm{Cv}$ positivo y el $\mathrm{Cv}$ negativo dentro del nivel Ef tristeza, y favorable al $\mathrm{Cv}$ negativo (tabla 1 ). Si bien también se mostraron diferencias parcialmente significativas $(p=.051)$ con un tamaño del efecto ligeramente moderado $(r=.021)$ entre el $\mathrm{Cv}$ positivo y el $\mathrm{Cv}$ neutro dentro del nivel de Ef triste, y favorable al Cv neutro. Estos resultados parecen indicar que las diferencias entre el Cv positivo y negativo solo se producen cuando la expresión facial es de tristeza.

Tabla 1

Diferencias en el reconocimiento de la expresión facial de alegría y tristeza dependiendo del tipo de información verbal (análisis de los efectos simples).

\begin{tabular}{|c|c|c|c|c|c|c|c|c|}
\hline \multirow[t]{2}{*}{$\mathrm{Cv}$} & \multicolumn{2}{|c|}{ Ef Alegría } & \multicolumn{2}{|c|}{ Ef Tristeza } & \multirow[t]{2}{*}{$M_{i-j}$} & \multirow[t]{2}{*}{$S E$} & \multirow[t]{2}{*}{$p$} & \multirow[t]{2}{*}{$T E$} \\
\hline & $M$ & $D T$ & $M$ & $D T$ & & & & \\
\hline Positivo & 1.96 & .20 & 1.64 & .56 & .320 & .083 & .000 & .233 \\
\hline Neutral & 1.88 &, 22 & 1.84 & .28 & .040 & .049 & .420 & .013 \\
\hline Negativo & 1.92 & .34 & 1.94 & .24 & -.020 & .061 & .773 & .002 \\
\hline \multirow[t]{3}{*}{ Ef } & \multicolumn{4}{|c|}{$\mathrm{Cv}$} & $M_{i-j}$ & $S E$ & $p$ & $T E$ \\
\hline & $M$ & $D T$ & $M$ & $D T$ & & & & \\
\hline & \multicolumn{2}{|c|}{ Positivo } & \multicolumn{2}{|c|}{ Neutral } & & & & \\
\hline Alegría & 1.96 & .20 & 1.88 & .22 & .080 & .039 & .133 & .080 \\
\hline \multirow[t]{2}{*}{ Tristeza } & 1.64 & .56 & 1.84 & .28 & -.200 & .081 & .051 & .111 \\
\hline & \multicolumn{2}{|c|}{ Positivo } & \multicolumn{2}{|c|}{ Negativo } & & & & \\
\hline Alegría & 1.96 & .20 & 1.92 & .34 & .040 & .057 & 1.00 & .010 \\
\hline \multirow[t]{2}{*}{ Tristeza } & 1.64 & .56 & 1.94 & .24 & -.300 & .087 & .003 & .196 \\
\hline & \multicolumn{2}{|c|}{ Neutral } & \multicolumn{2}{|c|}{ Negativo } & & & & \\
\hline Alegría & 1.88 & .22 & 1.92 & .34 & -.040 & .060 & 1.00 & .009 \\
\hline Tristeza & 1.84 & .28 & 1.94 & .24 & -.100 & .052 & .174 & .058 \\
\hline
\end{tabular}

Nota. Cv: Contexto Verbal; Ef: Expresión Facial.

La interpretación de los análisis de los efectos simples evidencia un deterioro en el reconocimiento de la expresión facial solo cuando la expresión de tristeza se asocia a un contexto verbal positivo, pudiéndose inferir un efecto de incongruencia entre la información verbal positiva y la expresión facial negativa podría estar perjudicando el reconocimiento posterior de dicha expresión. Sin embargo, este efecto de incongruencia no se produce cuando la información verbal es negativa y la expresión facial positiva (figura 1). 
Figura 1

Diferencias en la tasa de reconocimiento de la Expresión Facial (Ef) de alegría y tristeza respecto al Contexto Verbal $(\mathrm{Cv})\left({ }^{*} p<.01,{ }^{* *} p\right.$ $<.001$ ).

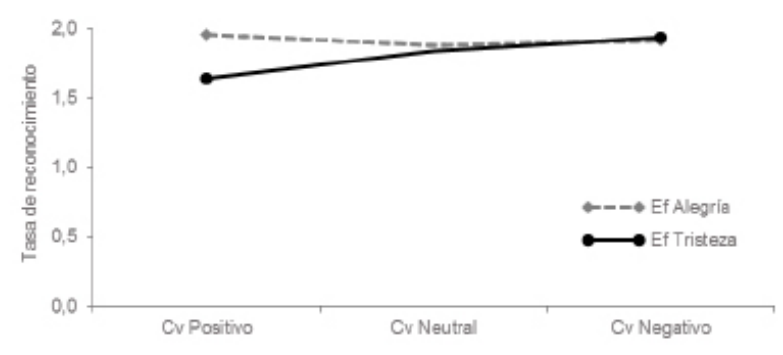

El ANOVA de medidas repetidas para el tiempo de respuesta no mostró efectos significativos de las variables $\mathrm{Cv}[F$ $\left.(2,98)=1.50, p=.227, \eta_{p}^{2}=.03, p=.31\right], \operatorname{Ef}[F(1,49)=2.75$, $\left.p=.104, \eta_{p}^{2}=.05, p=.37\right]$, ni de la interacción entre Cv y Ef $\left[F(2,98)=0.357, p=.701, \eta_{\mathrm{p}}^{2}=.01, P=.11\right]$.

\section{Discusión}

El paradigma utilizado ha permitido evidenciar el efecto que el contexto verbal referido a la vida de la persona productora de la expresión facial tiene sobre el reconocimiento posterior de la emoción expresada por dicha persona (alegría y tristeza). Estos resultados son relevantes en tanto hasta la fecha dicho efecto se había comprobado solo atendiendo a palabras o frases utilizadas como prime; sin embargo, los resultados obtenidos en la presente investigación evidencian que el conocimiento más general de la vida de las personas que expresan una emoción también afecta al reconocimiento de la expresión emocional a través de procesos automáticos (39 ms de exposición). Los resultados obtenidos permiten concluir que se produce un efecto negativo sobre la tasa de reconocimiento derivado de la incongruencia entre el $\mathrm{Cv}$ positivo y la Ef de tristeza. Extrapolando a la vida cotidiana quiere decir que cuando tenemos información positiva de una persona que está expresando tristeza se produciría un deterioro en la capacidad del perceptor para reconocer dicha expresión. Sin embargo, el efecto contrario no se ha producido, es decir, cuando tenemos información negativa de una persona que está expresando alegría no se produce un deterioro en el reconocimiento de dicha emoción. Quizá esto se deba a la elevada saliencia perceptiva de la sonrisa en la expresión facial, que facilitaría su reconocimiento con cierto grado de independencia respecto al contexto, hasta el punto de interpretar los ojos no alegres como alegres cuando se focaliza la sonrisa de manera previa a la valoración (Fernández, Avero y Gutiérrez, 2012).

Por otro lado, el que no se haya observado un efecto facilitador de la congruencia entre el $\mathrm{Cv}$ y la Ef, como así ha sido documentado para contextos verbales menos elaborados (véase Matsumoto y Hwang, 2010; Wieser y Brosch, 2012) puede ser debido a un posible efecto techo derivado de la elevada tasa media de reconocimiento emocional obtenida (véase figura
1). Estos resultados pueden interpretarse dentro del modelo constructivista (Barrett y Kensinger, 2010), donde la información referida a la expresión facial estaría facilitando el reconocimiento de la emoción expresada, incluso en mayor grado que la propia conformación de la musculatura facial (Barrett y Kensinger, 2010; Barrett et al., 2007). El efecto de incongruencia encontrado en la presente investigación podría estar evidenciando la fuerza ejercida por el Cv sobre la Ef, en este caso perjudicando el reconocimiento de una emoción que sea contraria a la valencia emocional del $\mathrm{Cv}$.

Estos resultados también se justifican dentro de los supuestos de los modelos de redes semánticas de la emoción (véase Niedenthal, 2008), que permiten entender cómo a partir de un conjunto de unidades semánticas se puede construir un nodo de información relativo a la vida de una persona que puede activarse y ejercer un efecto significativo sobre la valoración de, en este caso, una expresión facial emocional. De igual manera, la explicación tiene un escalón más a nivel de las estructuras neuronales que mantienen funciones comunes en el procesamiento de la emoción y el lenguaje (Badre et al., 2005; Gitelman et al., 2005; Wager et al., 2008), y que permiten comprender la estrecha relación entre contexto verbal y reconocimiento emocional.

Respecto a la automaticidad de este proceso, y a partir de la metodología planteada, se puede decir que, según los criterios de Martin y Levey (1978) para una respuesta de evaluación, se cumple que el proceso se da de manera inmediata, sin apenas esfuerzo, sin conciencia y sin que interfieran procesos cognitivos superiores. Sin embargo, se requiere realizar más estudios que permitan confirmar el supuesto de automaticidad, por ejemplo utilizando tareas concurrentes para confirmar que apenas consume recursos cognitivos, o tareas subliminales para confirmarían la no conciencia del proceso. La posible automatización del proceso implica que los efectos encontrados podrían producirse fuera de la consciencia. En ámbitos como el judicial, donde la expresividad emocional de los acusados tiene importantes implicaciones en las decisiones judiciales (e.g., Heath, 2009), la posibilidad de que el contexto verbal (e.g., la información vertida por los medios de comunicación sobre el acusado), pudiera influir de manera no deliberada sobre la interpretación que el jurado realiza de la emoción expresada por el acusado, podría implicar algún grado de influencia sobre las decisiones judiciales.

Por otro lado, este trabajo apoya el supuesto de que la información verbal tiene efectos importantes sobre el procesamiento de la información emocional, y que este efecto se mantiene conforme se incrementa el grado de elaboración de la información, desde las etiquetas verbales, pasando por las situaciones sociales (frases), hasta la información relativa a la vida de las personas. Se infiere la capacidad de integrar la información que tiene el cerebro, y que permite que información muy elaborada se active de manera integrada y automática, lo que mejoraría la adaptación al medio de las personas. 
Las limitaciones del trabajo se centran en el número de estímulos utilizados, dado que tan solo se utilizaron cuatro expresiones faciales diferentes en la primera fase (dos de hombre y dos de mujer). Esto responde a que el proceso de inducción del $\mathrm{Cv}$ relativo a la vida de una persona (fase I), requiere de un mayor tiempo de exposición respecto a la simple presentación de una palabra o una frase, que ha sido la forma habitual de generar Cv (véase Wieser y Brosch, 2012). En este experimento el tiempo de exposición del Cv se estimó en dos minutos, durante el cual el sujeto debía mirar la expresión facial y las palabras que aparecían rodeándola, atendiendo a la instrucción de que posteriormente se le harían algunas preguntas al respecto.

De esta forma se pretendía integrar la información verbal con la expresión facial, para promover el efecto sobre el reconocimiento en la fase II. Si se pudiera reducir el tiempo de exposición en esta fase sin detrimento de la sensibilidad de la prueba, lo idóneo sería incluir más díadas Cv-Ef, que ampliaran el rango de los valores obtenidos. Además de incrementar el número de estímulos habría que ajustar en mayor grado los porcentajes de alegría y tristeza asignados a las expresiones con morphing, de forma que sean más sutiles y por lo tanto se incremente la dificultad de la tarea que junto al incremento del número de estímulos, evitaría el efecto techo que posiblemente ha perjudicado la presencia de los efectos esperados en la congruencia entre Cv y Ef. También sería importante equilibrar las valoraciones realizadas al $\mathrm{Cv}$ a través de pruebas piloto que permita la adecuada elección de las palabras que conforman los niveles del $\mathrm{Cv}$, de forma que se establezca la misma distancia en la valoración del $\mathrm{Cv}$ positivo y negativo respecto al neutro. Por último, algunas limitaciones centradas en la muestra, como el hecho de que haya sido obtenida de la población universitaria y que la mayoría sean mujeres, lo que estaría perjudicando la posible generalización de los resultados.

Futuros trabajos deberían investigar variaciones importantes del paradigma, como el tipo de $\mathrm{Cv}$ y expresión emocional utilizados. En el presente trabajo se utilizaron $\mathrm{Cv}$ positivos, negativos y neutros, así como expresiones mixtas de alegría y tristeza, pero la metodología permite utilizar $\mathrm{Cv}$ verbales específicos de cada emoción básica, a partir de la selección de palabras congruentes con dichas emociones. Por otro lado, sería necesario elaborar una base baremada del binomio $\mathrm{Cv}$-Ef, que permita su utilización de manera más controlada, atendiendo a los niveles de valencia, activación y control, en el mismo sentido que las dimensiones utilizadas en los trabajos de Lang, Bradley y Cuthbert (1999), para las fotografías de contenido emocional (IAPS: Internacional Affective Picture System). Por último, también sería de interés su aplicación a población clínica, para analizar como el $\mathrm{Cv}$ puede modular los procesos de percepción en trastornos como el autismo o el Alzheimer. En este sentido, resultaría de gran valor terapéutico el comprender cómo el contexto verbal podría facilitar el reconocimiento de las expresiones faciales emocionales, con el fin de implementar procedimientos específicos en los programas de rehabilitación.

\section{Referencias}

1. Aviezer, H., Hassin, R. R., Ryan, J., Grady, C., Susskind, J., Anderson, A., Moscovitch, M. \& Bentin, S. (2008). Angry, disgusted, or afraid? Studies on the malleability of emotion perception. Psychological Science, 19, 724-732. http:// dx.doi.org/10.1111/j.1467-9280.2008.02148.x

2. Aviezer, H., Bentin, S., Dudarev, V. \& Hassin, R. R. (2011). The automaticity of emocional face-context integration. Emotion, 11, 1406-1414. http://dx.doi.org/10.1037/ $\underline{\mathrm{a} 0023578}$

3. Badre, D., Poldrack, R. A., Paré-Bagoev, E. J., Isler, R. Z. \& Wagner, A. D. (2005). Dissociable controlled retrieval and generalized selection mechanisms in ventrolateral prefrontal cortex. Neuron, 47, 907-918.

4. Barrett, L. F. \& Kensinger, A. K. (2010). Context is routinely encoded during emotion perception. Psychological Science, 21, 595-599. http://dx.doi.org/10.1177/0956797610363547

5. Barrett, L.F., Lindquist, K. \& Gendron, M. (2007). Language as a context for the perception of emotion. Trends in Cognitive Sciences, 11, 327-332. http://dx.doi. org/10.1016/j.tics.2007.06.003

6. Barret, L. F., Pesquita, B. \& Gendron, M. (2011). Context in emotion perception. Current Directions in Psychological Science, 20, 286. http://dx.doi. org/10.1177/0963721411422522

7. Bruner, J. (1957). On perceptual readiness. Psychological Review, 64, 123-152. http://dx.doi.org/10.1037/h0043805

8. Bruner, J. S., Postman, L. \& Rodrigues, J. (1951). Expectation and the perception of color. The American Journal of Psychology, 64, 216-227. http://dx.doi. org/10.2307/1418668

9. Ekman, P. \& Cordaro, D. (2011). What is meant by calling emotions basic. Emotion Review, 3, 364-370. http://dx.doi. org/10.1177/1754073911410740

10. Fernández, A., Avero, P. \& Gutiérrez, M. (2012). Una sonrisa en la boca hace que los ojos parezcan alegres. Escritos de Psicología, 5, 25-33. http://dx.doi.org/10.5231/psy. writ.2012.0202

11. Gitelman, D. R., Nobre, A. C., Sonty, S., Parrish, T. B. \& Mesulam, M. M. (2005). Language network specializations: An analysis with parallel task designs and functional magnetic resonance imaging. Neuroimage, 26, 975-985. http://dx.doi.org/10.1016/j.neuroimage.2005.03.014

12. Gordillo, F., Lozano, J. H., López, R. M., Pérez, M. A., Arana, J. M. \& Mestas, L. (2013). Emoción, contexto verbal y percepción de la expresión facial: Dime quién eres y te diré cómo te percibo. Ansiedad \& Estrés, 19, 131147.

13. Hassin, R. R., Aviecer, H. \& Bentin, S. (2013). Inherently ambiguous: Facial expressions of emotions, in context. Emotion Review, 5, 60-65. http://dx.doi. org/10.1177/1754073912451331

14. Heath, W. P. (2009). Arresting and convicting the innocent: the potential role of an "inappropriate" emotional display in 
the accused. Behavioral Sciences \& the Law, 27, 313-332. http://dx.doi.org/10.1002/bsl.864

15. iTinysoft (2002). Magic Morph (versión 1.95B). Disponible en www.etinysoft.com.

16. Jack, R. E., Garrod, O. G. B., Yu, H., Caldara, R. \& Schyns, P. G. (2012). Facial expressions of emotion are not culturally universal. Proceedings of the National Academy of Sciences of the United States of America, 109, 7241-7244. http://dx.doi.org/10.1073/pnas.1200155109

17. Jack, R. E., Garrod, O. G. B. \& Schyns, P. G. (2014). Dynamic facial expressions of emotion transmit an evolving hierarchy of signals over time. Current Biology, 24, 187-192. http://dx.doi.org/10.1016/j.cub.2013.11.064

18. Janiszewski, C. \& Wyer, R. S. (2014). Content and process priming: A review. Journal of Consumer Psychology 24, 96-118. http://dx.doi.org/10.1016/j.jcps.2013.05.006

19. Kim, H., Somerville, L. H., Johnstone, T., Polis, S., Alexander, A.L., Shin, L. M. \& Whalen, P. J. (2004). Contextual modulation of amygdala responsivity to surprised faces. Journal of Cognitive Neuroscience, 16, 1730-1745. http:// dx.doi.org/10.1162/0898929042947865

20. Lang, P. J., Bradley, M. M. \& Cuthbert, B. N. (1999). International affective picture system (IAPS): Technical manual and affective ratings. Gainesville, FL: The Center for Research in Psychophysiology, University of Florida.

21. Lindquist, K. A., Barrett, L. F., Bliss-Moreau, E. \& Russell, J. A. (2006). Language and the perception of emotion. Emotion 6, 125-138. http://dx.doi.org/10.1037/1528-3542.6.1.125

22. Martin, I. \& Levey, A. B. (1978). Evaluative conditioning. Advances in Behavior Research \& Therapy, 1, 57-102. http://dx.doi.org/10.1016/0146-6402(78)90013-9

23. Matsumoto, D. \& Hwang, H. (2010). Judging face in context. Social and Personality Psychology Compass, 3, 1-10. http://dx.doi.org/10.1111/j.1751-9004.2010.00271.x

24. Niedenthal, P. M. (2008). Emotion concepts. En M. Lewis, J. M. Haviland-Jones, \& L. F. Barrett (Eds.), The handbook of emotion (pp. 597-600) ( $3^{\text {rd }}$ ed.). New York: Guilford Press.

25. Pell, M. D., Jaywant, A., Montea, L. \& Kotz, S. A. (2012). Emotional speech processing: Disentangling the effects of prosody and semantic cues. Cognition and Emotion, 25, 834-853. http://dx.doi.org/10.1 $\underline{080 / 02699931.2010 .516915}$

26. Redondo, J., Fraga, I., Comesaña, M. \& Perea, M. (2005). Estudio normativo de 478 palabras españolas. Psicológica, 26, 317-326.

27. Righart, R. \& de Gelder, B. (2008). Rapid influence of emotional scenes on encoding of facial expressions. An ERP study. Social Cognitive and Affective Neuroscience, 3, 270278. http://dx.doi.org/10.1093/scan/nsn021
28. Russell, J. A. (1994). Is there universal recognition of emotion from facial expression? A review of the cross-cultural studies. Psychological Bulletin, 115, 102-141. http://dx.doi. org/10.1080/02699930902809375

29. Russell, J. A. (2003). Core affect and the psychological construction of emotion. Psychological Review, 110, 145172. http://dx.doi.org/10.1037/0033-295X.110.1.145

30. Russell, J. A., Bachorowski, J. A. \& Fernández-Dols, J. M. (2003). Facial and vocal expressions of emotion. Annual review of psychology, 54, 329-349. http://dx.doi. org/10.1146/annurev.psych.54.101601.145102

31. Schwarz, K. A., Wieser, M. J., Gerdes, A. B. M., Mühlberger, A. \& Pauli, P. (2013). Why are you looking like that? How the context influences evaluation and processing of human faces. SCAN, 8, 438-445. http://dx.doi.org/10.1093/ $\underline{\text { scan } / \mathrm{nss} 013}$

32. Shen-Mou, H. \& Lee-Xieng, Y. (2013). Sequential effects in facial expression categorization. Emotion, 13, 573-586. http://dx.doi.org/10.1037/a0027285

33. Schneider, W., Eschman, A. \& Zuccolotto, A. (2002). E-Prime User's Guide. Pittsburg: Psychology Software Tools Inc.

34. Tottenham, N., Tanaka, J.W., Leon, A.C., McCarry, T., Nurse, M., Hare, T.A., Marcus, D.J., Westerlund, A., Casey, B.J. \& Nelson, C. (2009). The NimStim set of facial expressions: Judgments from untrained research participants. Psychiatry Research, 168, 242-249. http://dx.doi. org/10.1016/j.psychres.2008.05.006

35. Wager, T., Barrett, L. F., Bliss-Moreau, E., Lindquist, K. A., Duncan, S., Kober, H., Joseph, J., Davidson, M. \& Mize, J. (2008). The neuroimaging of emotion. En: M. Lewis, J. M. Haviland-Jones \& L. F. Barrett (Eds.), The Handbook of Emotion (pp 249-279) ( $3^{\text {rd }}$ ed.). Guilford Press.

36. Wieser, M. J. \& Brosch, T (2012). Faces in context: A review and systematization of contextual influences on affective face processing. Frontiers in Psycholgy, 3, 471. http://dx.doi.org/10.3389/fpsyg.2012.00471

Fecha de recepción: 5 de septiembre, 2015 Fecha de recepción de la versión modificada: 6 de noviembre, 2015 Fecha de aceptación: 22 de diciembre, 2015 
FERNANDO GORDILLO, MIGUEL ÁNGEL PÉREZ, GABRIELA CASTILLO, LILIA MESTAS, JOSÉ M. ARANA, RAFAEL MANUEL LÓPEZ

Anexo 1

Tipo de palabra, atendiendo a la valencia (Val) y a la activación (Act), y tipo de expresión facial utilizadas en el experimento.

\begin{tabular}{|c|c|c|c|c|c|c|c|}
\hline \multicolumn{4}{|c|}{$\begin{array}{l}\text { Palabras contenido emocional } \\
\text { (Redondo et al., 2005) }\end{array}$} & \multicolumn{4}{|c|}{$\begin{array}{l}\text { NimStimFace Stimulus Set } \\
\text { (Tottenham et al., 2009) }\end{array}$} \\
\hline Palabra & Val (Act) & Palabra & Palabra & Expresión & Género & Tipo & Boca \\
\hline Familia & $7.64(4,41)$ & Maestro & $5.39(4,36)$ & 03F-HA-C & Mujer & Alegría & Cerrada \\
\hline Ilusión & $7.79(5,49)$ & Materia & $4.90(3,38)$ & 03F-SA-C & Mujer & Tristeza & Cerrada \\
\hline Aventura & $7.42(6,56)$ & Litro & $5.04(3,08)$ & 07F-HA-C & Mujer & Alegría & Cerrada \\
\hline Sexo & $7.67(7,02)$ & Oficina & $4.93(3,84)$ & 07F-SA-C & Mujer & Tristeza & Cerrada \\
\hline Optimismo & $7.60(4,74)$ & Propietario & $5.18(3,13)$ & 24M-HA-C & Hombre & Alegría & Cerrada \\
\hline Abuso & $1.72(7,41)$ & Ingenuidad & $5.20(3,94)$ & 24M-SA-C & Hombre & Tristeza & Cerrada \\
\hline Pobreza & $1,87(6,86)$ & Década & $5.09(3,67)$ & 25M-HA-C & Hombre & Alegría & Cerrada \\
\hline Horror & $2.17(7,36)$ & Redacción & $5.26(3,69)$ & 25M-SA-C & Hombre & Tristeza & Cerrada \\
\hline Pesadilla & $1.93(7,43)$ & Motor & $5.14(3,67)$ & & & & \\
\hline Depresión & $1.59(6,80)$ & Despacho & $5.01(4,14)$ & & & & \\
\hline
\end{tabular}

Anexo 2

Tipos de contexto verbal utilizados en la fase I a partir de las palabras seleccionadas y expresiones mixtas utilizadas (50\% alegría / 50\% tristeza).

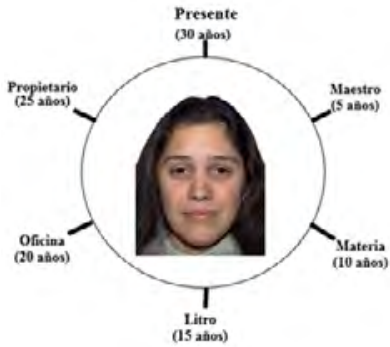

Cv_Neutral 1

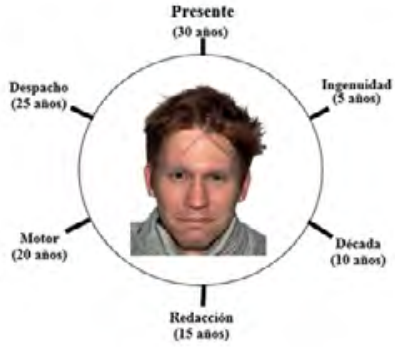

Cv_Neutral 2

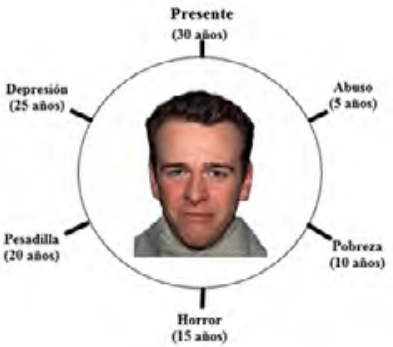

Cv_Negativo

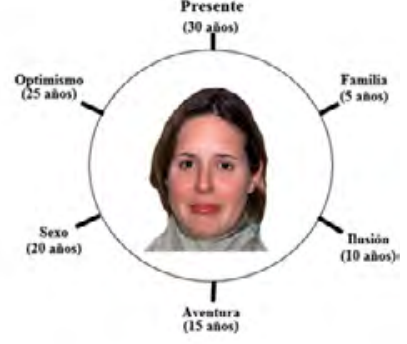

Cv_Positivo 